\title{
Drug-induced (poly I:C) pyrexic responses: Congruence with a compensatory conditioning analysis
}

\author{
DENNIS G. DYCK and TIMOTHY A. G. OSACHUK \\ University of Manitoba, Winnipeg, Manitoba, Canada \\ and \\ ARNOLD H. GREENBERG \\ Manitoba Institute of Cell Biology, Winnipeg, Manitoba, Canada
}

\begin{abstract}
Preliminary experiments indicated that DBA/2J mice exhibit a pyrexic response 4-6 h after injection with the synthetic, double-stranded polynucleotide, polyinosinic polycytidylic acid (poly I:C), a macrophage and natural killer (NK) cell activating compound. We tested the hypothesis that a procedure involving repeated paired delivery of odor cues and poly $I: C$ would result in the elicitation of a conditioned "drug-compensatory" hypothermic response to a cued placebo injection. A $2 \times 2$ factorial design was employed in a two-phase experiment with mice. In the first phase, two groups of mice were given four weekly, signaled injections of either poly I:C (50 $\mu \mathrm{g} / \mathrm{mouse})$ or saline. Rectal temperature was taken immediately prior to the injection and at 1-h intervals for the next $6 \mathrm{~h}$. During the second 4-week phase, half of the mice in the saline (S) and poly I:C (P) groups were maintained on the same drug regimen (S-S; P.P); the other half were shifted to the opposite drug condition (P-S; S-P). The results indicated that the mice given paired cue-drug delivery in Phase 1 demonstrated a robust hypothermic response to cued placebo injections in Phase 2 (Group P-S). This drug-compensatory hypothermic response was evident over all 4 weeks of testing. These results are consistent with the interpretation that drug-compensatory conditioning occurred to cues paired with poly I:C, and, more generally, our findings support the hypothesis that the effects of this immunostimulatory agent are subject to modificaton by the central nervous system.
\end{abstract}

Following repeated, signaled administration of the synthetic nucleic acid polymer, polyinosinic polycytidylic acid (poly I:C), there is an attenuation of the drug's immunostimulatory effects on natural killer (NK) cell activation. This effect is, at least in part, an associative consequence (Dyck, Greenberg, \& Osachuk, 1986). The tolerance effect is reversible by decremental conditioning procedures such as extinction and CS preexposure (Dyck et al., 1986), and is influenced by drug-context manipulations (Dyck, Driedger, Nemeth, Osachuk, \& Greenberg, 1987).

The observations of the tolerance effect to date are congruent with a Pavlovian conditioning hypothesis of tolerance (Siegel, 1978). This hypothesis postulates that en-

This research was jointly supported by grants from the Natural Sciences and Engineering Research Council of Canada (Grant A9941), the U.S.P.H.S. (Grant MH43778-01), the R. J. R. Nabisco Corporation, and the Manitoba Mental Health Research Foundation. A.H.G. is a Terry Fox Cancer Research Scientist of the National Cancer Institute of Canada. We thank John R. Wilson and Linda Wilson for lending us their BAT12 digital thermometer and probe for the preliminary experiments and for helpful discussions in the design and measurement aspects of these experiments. Correspondence concerning this article should be addressed to Dennis G. Dyck, Psychology Department, University of Manitoba, Winnipeg, Manitoba R3T 2N2, Canada. vironmental cues paired with poly I:C elicit drugcompensatory conditional responses (CRs) that oppose and diminish the effects of the drug. Such an analysis predicts more pronounced tolerance when drug-experienced animals are given the drug in the presence, rather than in the absence, of environmental drug-signaling cues. Furthermore, this hypothesis predicts that when drugsignaling cues are not followed by the usual pharmacological consequences, a CR opposite to the drug effect should be observed.

Although the research to date is congruent with a compensatory conditioning model, such an interpretation is not exclusive, and the evidence can just as easily be accommodated by models that do not require a compensatory response mechanism (e.g., Baker \& Tiffany, 1985; Paletta \& Wagner, 1986). Since the role of conditioning in the NK cell tolerance effect has been measured only indirectly (i.e., on the basis of an altered response to the drug; a UR), the data from such experiments do not unequivocally support a compensatory conditioning model. The critical observation for this interpretation is that the compensatory CR must be directly observed, and to date such an observation has not been made in the NK cell tolerance work. In part, this difficulty reflects a measurement problem. That is, resting levels of NK cell ac- 
tivity are already low in the DBA/2J mouse, and it is therefore difficult to detect further decreases which would reflect a compensatory $\mathrm{CR}$ to predrug cues.

In the present study, we evaluated the conditionability of a different, but related, response to the drug, namely, a thermic response. Compensatory thermic responses have been successfully conditioned with other drugs, including morphine (e.g., Siegel, 1978), so it seemed reasonable that such a response might provide a sensitive index of compensatory conditioning to poly I:C.

Apart from the foregoing measurement issue, an important consideration in choosing to study the thermic effects of poly I:C, an immunomodulatory agent, was to evaluate the possibility that such effects are centrally mediated. Successful conditioning of thermic effects would then assist in defining a neuroimmunoregulatory pathway. Although pyrexic effects to poly I:C have not, to our knowledge, previously been reported, this macrophageactivating compound is known to stimulate the release of several monokines that are known to increase pyrexic responses through their effects on the central nervous system. These include interleukin-1 (IL-1) (Dinarello, Conti, $\&$ Weir, 1986) and tumor necrosis factor (TNF- $\alpha$ ) (Dinarello, Cannon, et al., 1986). IL-1, which is of particular interest because of its effect on immunosuppressive neuroendocrine hormones (Besedovsky, del Rey, Sorkin, \& Dinarello, 1986), is a small molecular-weight peptide that increases body temperature. The mechanism by which IL-1 raises body temperature is not well understood, but it is thought to act on the brain's central thermoregulatory set point within the hypothalamus by the action of some secondary agent, likely prostaglandin E2 (Dinarello, 1984). Although the source of any observed pyrexic responses to poly I: $C$ would require independent verification, for reasons to be elaborated in the discussion, we hypothesize that such effects occur via the release of IL-1 from stimulated macrophages.

The present study, then, was designed to evaluate further the hypothesis that the pyrexic effects of poly I:C, like its immunological effects, might be centrally mediated. The strategy to evaluate this hypothesis involved an attempt to condition the pyrexic effects of poly I:C to environmental cues paired with the drug. Specifically, we asked whether a pyrexic response to poly I:C could be observed, whether such a response could be conditionable, and whether the direction of the conditioned response would be consistent with a compensatory conditioning analysis.

\section{EXPERIMENT 1: POLY I:C-INDUCED PYREXIA}

In two separate preliminary experiments, we measured the effect of various doses of a single injection of poly I:C on rectal temperature. A BAT 12 digital thermometer and Bailey probe (Model IT- $1, .025$ in. diameter) were used to monitor temperature changes in mice that had been maintained at ambient temperature. The probe was in- serted rectally to a depth of $1-2 \mathrm{~cm}$. Temperature was measured immediately before and after i.p. injection of the drug, at 30 -min intervals for the next $2 \mathrm{~h}$, and at hourly intervals for another $4 \mathrm{~h}$. The results of this experiment are shown in Figure 1.

Although temperature increases above preinjection levels were difficult to detect, a distinct increase in body temperature was seen in treated animals, relative to salinecontrol animals, 4-6 h after injection with either $20 \mu \mathrm{g}$ or $100 \mu \mathrm{g}$ of poly I:C (Experiment 1A). Furthermore, in contrast to the delayed effect of poly I:C on temperature, the effect of exogenous IL- 1 was rapid, with a noticeable peak in temperature at approximately $1 \mathrm{~h}$ after injection. These results provide preliminary evidence for a pyrexic response to poly I:C.

In a second preliminary experiment (Experiment 1B), temperature was monitored in four groups of mice. These subjects were exposed to the temperature-assessment procedure on Day 1; on Day 2, a saline injection was followed by temperature assessment. On Day 3, the mice were injected with either saline or 10,20 , or $50 \mu \mathrm{g}$ of poly I:C. Because of our interest in conditioning, we were interested in determining whether pyrexic effects would be observable under the relatively short adaptation period.

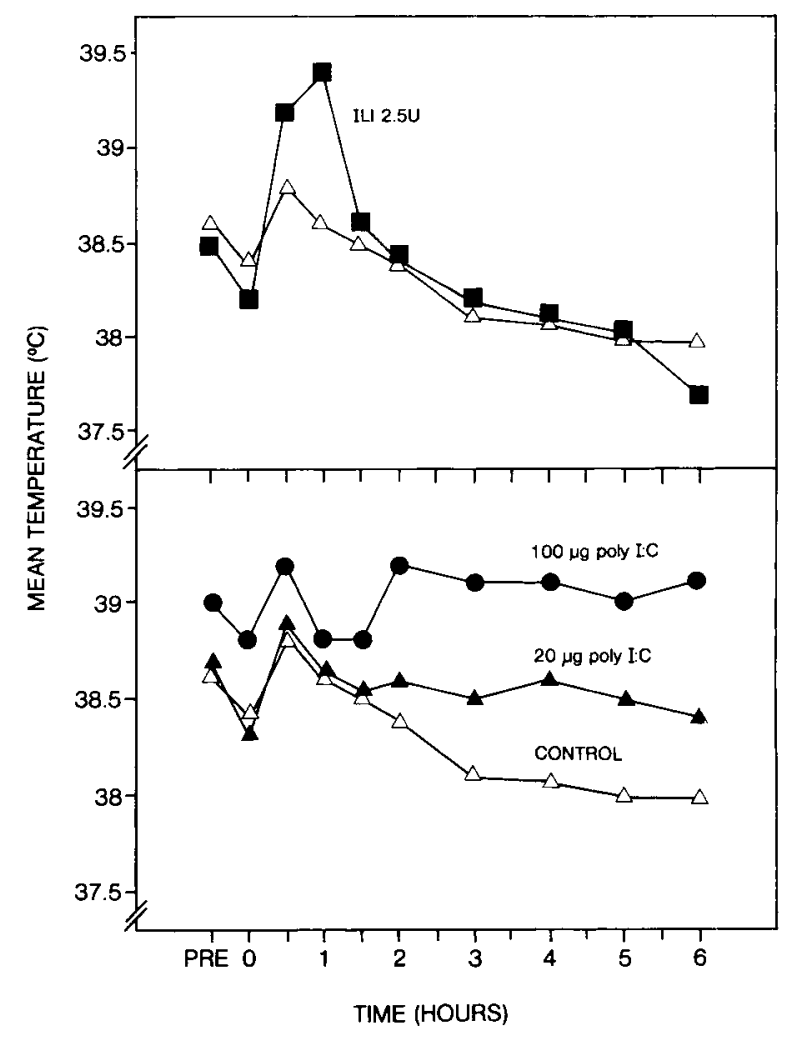

Figure 1. Febrile response of mice to a single intraperitoneal injection of $2.5 \mathrm{U}$ of interleukin-1 ( $\mathrm{E}$ ) (upper panel), $100 \mu \mathrm{g}$ of poly I:C $(\bullet)$, or $20 \mu$ g of poly I:C ( $\Delta$ ) (lower panel) compared to salineinjected controls $(\triangle)$. All animals were adapted to the temperatureassessment procedure for 6 days prior to the initiation of the experiment. 


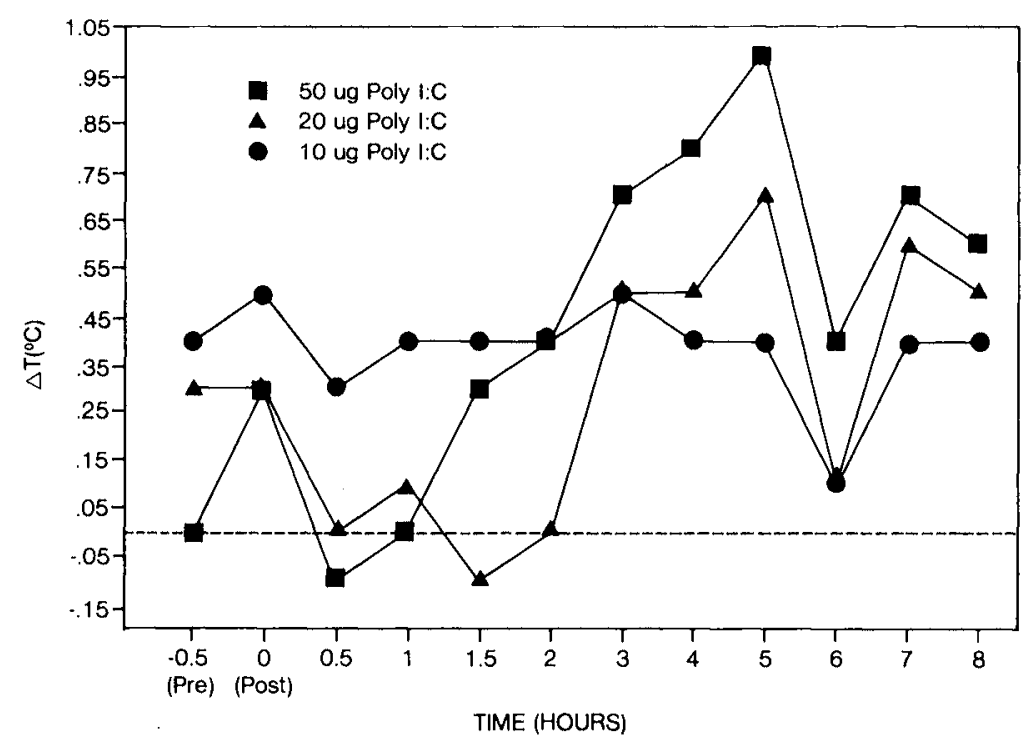

Figure 2. Fever induced by the intraperitoneal injection of poly I:C. In contrast to the previous experiment, mice had no prior handling before drug administration. Temperature scores are expressed as the difference $(\Delta)$ between poly I:C-injected and saline-injected (control) mice.

Specifically, our concern was that extensive adaptation could result in the attenuation of conditioning through a CS preexposure effect (e.g., Dyck et al., 1986). The data are expressed as delta scores by subtracting the mean temperature of the control group from the temperature of each animal in each of the other groups at each repeated measurement interval. ${ }^{1}$ The results, which are shown in Figure 2, indicate that $50 \mu \mathrm{g}$ of poly I:C produced the most pronounced temperature elevation, expressed as a change from saline levels. Again, this change occurred 4-6 $\mathrm{h}$ after injection.

Although the cell sizes in these preliminary pilot studies were insufficient to document the temperature changes statistically, taken together, the results were sufficiently encouraging to attempt to condition these drug-induced thermic alterations to cues paired with poly I:C.

\section{EXPERIMENT 2: CONDITIONING OF POLY I:C-INDUCED PYREXIA}

In the present two-phase experiment, four groups of mice were exposed to repeated weekly signaled injections of poly I:C. In each phase, the mice received signaled injections on a weekly basis for 4 weeks followed by assessment of rectal temperature. The four groups of mice received $50 \mu \mathrm{g}$ of poly I:C immediately after exposure to odor cues in the first (P-S), second (S-P), neither (S-S), or both (P-P) phases of the experiment. On the basis of a compensatory conditioning analysis, we predicted that Group P-S would show a hypothermic response to cued placebo injections, relative to control animals. Further- more, given the preliminary observation that the pyrexic effect of the drug was somewhat delayed, we anticipated that the compensatory hypothermic response might also be most robust at the later measurement intervals. Finally, since the four cued-placebo injections for Group P-S represented unreinforced presentations of the CS, we predicted that the hypothesized hypothermic CR would show some evidence of extinction.

\section{Method}

\section{Subjects}

The subjects were 23 experimentally naive, 35 -day-old DBA/2J mice (Jackson Laboratories, Bar Harbor, ME). The mice were housed in groups of $\mathbf{4}$ in standard polypropylene cages with filter bonnets, and were maintained on a 12-h light cycle. Food and water were removed $1 \mathrm{~h}$ before each experimental session for the duration of the session. Cage cleaning and replacement of food and water occurred weekly and were coordinated with the weekly experimental protocol. The mice otherwise remained undisturbed between injections.

\section{Procedure}

Two weeks after arrival of the mice in the laboratory, the mice were earpunched for identification. The experiment proper began one week later. Two groups of mice were given four weekly, cued injections of either $50 \mu \mathrm{g} /$ mouse of poly I:C (Group P) or $100 \mu \mathrm{l}$ of sterile Hanks Balanced Salt Solution (Group S). In the second phase, four additional weekly, cued injections were given such that half of the mice in each group received the same drug treatment as in Phase 1 (Groups P-P and S-S), and the other half were shifted to the opposite treatment (Groups P-S and S-P).

Conditioning and temperature assessment sessions began by moving the animals to the experimental room shortly after the onset of the light cycle, which began at $0700 \mathrm{~h}$. Food and water were 
removed at this time. Approximately $1 \mathrm{~h}$ later, individual mice were removed from their cages and placed in a box $(28 \times 17 \times 12 \mathrm{~cm})$ that contained $2 \mathrm{ml}$ of peppermint extract spread over $300 \mathrm{cc}$ of absorbent bedding weighing about $50 \mathrm{~g}$. A stopwatch was started after all mice were in the peppermint box. After 5 min in this distinctive environment, individual mice were picked up at random, swabbed with alcohol, and given an i.p. injection of either sterile Hanks Balanced Salt Solution or $50 \mu \mathrm{g} /$ mouse of poly I:C. Syringes were prepared in sterile fashion and a fresh needle was used for each mouse. Temperature was recorded immediately after injection with a BAT 12 digital thermometer and Bailey probe (Model IT-1). This procedure was repeated until all mice in a cage had been injected and had received an initial temperature reading. The order of running groups was consistent throughout the experiment. Temperature assessments were then taken at six hourly intervals and, following the last assessment, mice were transferred to a fresh cage with food and water, and were taken back to the colony room.

\section{Results}

Analyses were performed on both the raw temperature data and on the transformed data for ease of interpretation. As in the preliminary experiments, the latter data were expressed as delta scores by subtracting the mean temperature of the saline group at each measurement interval from individual subjects' temperature readings at that interval. Since the two measures produced virtually identical outcomes, the presentation of the results will focus on the transformed data, followed by a brief overview of the raw data.

\section{Analysis of Delta Data}

Phase 1. The results of Phase 1 are shown in Figure 3. Baseline temperature as reflected by the measure immediately following drug injection was assessed by a threeweek, repeated-measures analysis of variance (ANOVA). The fourth week's temperature data could not be collected due to a breakdown of the ventilation system and an increase in the ambient room temperature. This analysis did not yield a significant effect.

The temperature data for the six hourly readings following injection were analyzed with a three-way, repeated-measures ANOVA with weeks (3), blocks (3), and hours within blocks (2) as within-group factors. This analysis revealed a significant main effect for blocks $[F(2,22)=4.04, p<.05]$ and for hour $[F(1,11)=$ $11.88, p<.01]$. These effects, which indicate a significant drug effect, were qualified by a three-way interaction $[F(4,44)=4.69, p<.01]$. A breakdown of the interaction with Scheffe's procedure (Kirk, 1968) indicated that a significant increase in temperature was observed from the first to the second hour on all three weeks on Block 1. However, tolerance to the pyrexic effects of poly I:C observed at that temperature on both the first and the second hours of Block 1 was lower on Week 3 than on Week 1 (all $p s<.05$ ). Moreover, tolerance was also evident on the second block (the third and fourth readings), as revealed by lower temperature readings on the second

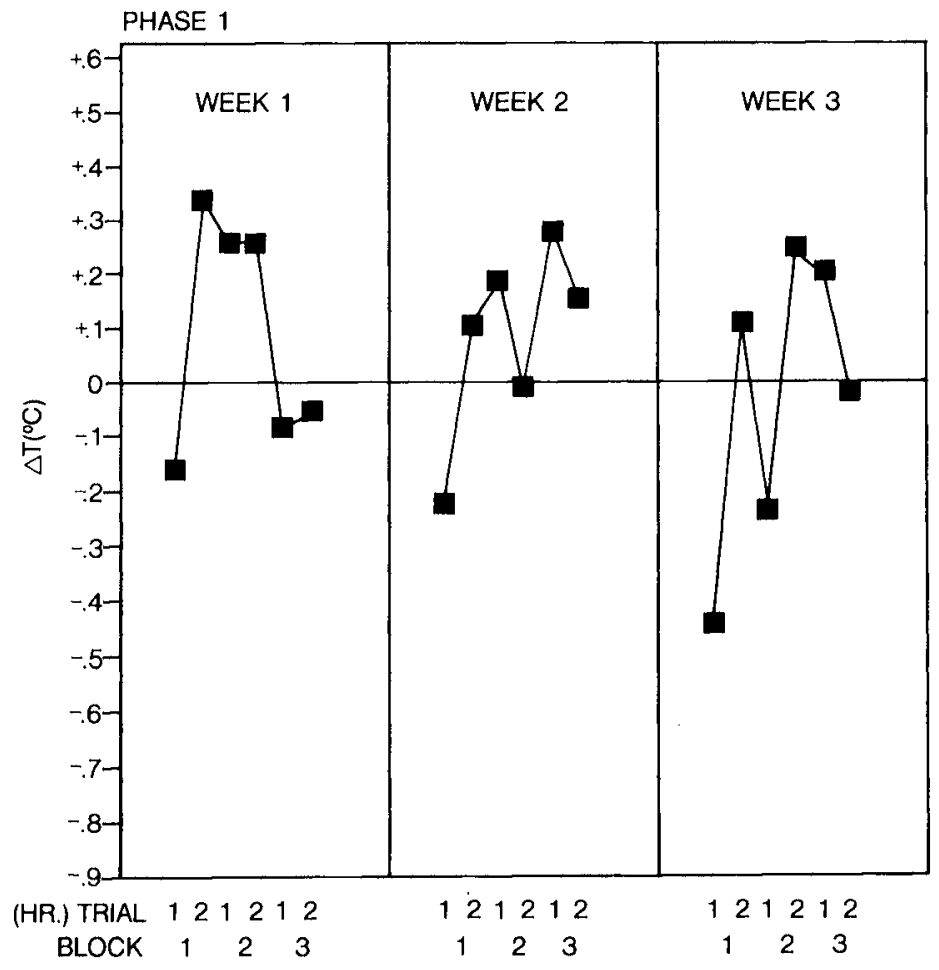

Figure 3. Phase 1 of the conditioning trials expressed as delta scores. Mice were exposed once weekly to signaled injections of either poly $\mathrm{I}: \mathrm{C}$ or saline. Results are expressed as in Figure 2. 


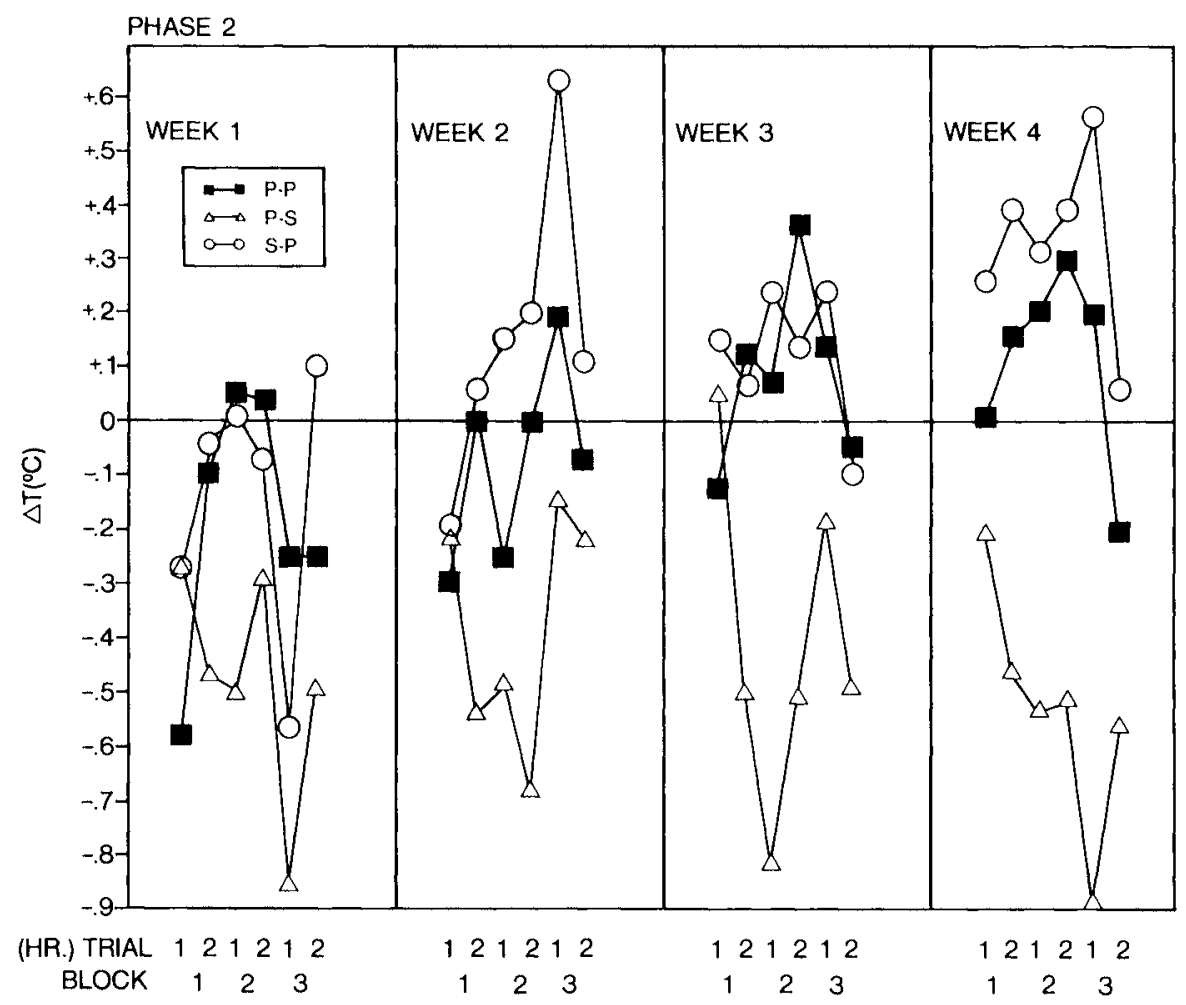

Figure 4. Phase 2 of the conditioning trials expressed as delta scores. Mice that had been given weekly cued injections of poly 1:C (P) or saline (S) in the first phase were given additional weekly cued injections, for 4 weeks, with half the mice in each group shifted to the opposite treatment, P-S $(\triangle)$ and S-P $(O)$. One group received only poly I:C, P-P $(\square)$. All results were compared with those of animals that received saline throughout the experiment and scored as the difference in temperature.

and third weeks, relative to the first week. Finally, by the last time-block, temperature had returned close to the baseline, and this did not differ as a function of weeks. These results, then, indicate the presence of a modest pyrexic response that is most visible 2-4 h postinjection. In addition, the decrease in peak temperature across weeks provides some evidence of tolerance to the pyrexic effects of the drug. However, in view of the modest pyrexic effects observed, this evidence for tolerance should not be overemphasized.

Phase 2. The baseline temperatures of the three experimental groups, expressed as delta scores, were analyzed with a mixed-design, repeated-measures ANOVA, with group as a between-groups factor (Groups P-P, P-S, and S-P) and weeks (4) as a within-group factor. No significant effects were found, which indicates that baseline temperature was stable and did not differ across groups.

The analysis of postinjection temperature utilized a mixed-design, repeated-measures ANOVA, with group as a between-groups factor (Groups P-P, P-S, and S-P) and weeks (4), blocks (3), and hours (2) as within-group factors. The only significant main effect in this analysis was that of weeks, which simply indicated that temperature decreased over the 4 weeks of Phase $2[F(3,45)=$ $5.59, p<.01]$. However, the importance of this main effect is overriden by the four-way interaction, which was significant $[F(12,90)=2.98, p<.01]$. The temperature changes of the various groups in Phase 2 are depicted in Figure 4 and convey the essential features of this interaction.

A breakdown of the interaction using Scheffé's correction procedure revealed the following theoretically significant effects: (1) on the first block, only Group P-S demonstrated a significant decline in body temperature from the first to the second hour ( $p s<.05$ at weeks 2 , 3 , and 4); (2) on the second block, Group P-S exhibited lower body temperatures than both of the remaining groups on all four weeks ( $p s<.05)$; and (3) Group S-P had a higher rectal temperature at the fifth measurementinterval (Block 3, Trial 1) than did Group P-P on Weeks 2 and 4. The first two observations constitute evidence of a robust conditioned hypothermic effect among drug-experienced animals reexposed to drug-signaling cues. This effect, however, did not show any evidence of diminishing over weeks (i.e., extinction). The observation of a somewhat higher temperature among Group S-P, relative to Group P-P, is consistent with the attenuation of tolerance of NK cell activation by CS preexposure (Dyck et al., 1986). On the other hand, neither Group P-P nor Group S-P gave evidence of reduced temperatures (i.e., tolerance) over the successive weeks of Phase 2. Thus, only the Phase 1 results provided some 
Table 1

\begin{tabular}{|c|c|c|c|c|c|c|c|c|c|c|c|c|}
\hline \multirow[b]{4}{*}{ Group } & \multicolumn{12}{|c|}{$\begin{array}{l}\text { Group Mean Temperature } \pm \text { Standard Error of the } \\
\text { Mean for Experiment 2, Phase } 1\end{array}$} \\
\hline & \multicolumn{12}{|c|}{ Hour } \\
\hline & \multicolumn{2}{|c|}{1} & \multicolumn{2}{|c|}{2} & \multicolumn{2}{|c|}{3} & \multicolumn{2}{|c|}{4} & \multicolumn{2}{|c|}{5} & \multicolumn{2}{|c|}{6} \\
\hline & $M$ & $S E M$ & $M$ & $S E M$ & $M$ & $S E M$ & $M$ & SEM & $M$ & SEM & $M$ & $S E M$ \\
\hline \multicolumn{13}{|c|}{ Week 1} \\
\hline $\mathbf{P}$ & 38.5 & .1 & 38.7 & .2 & 38.4 & .2 & 38.4 & .2 & 38.1 & .2 & 37.8 & .2 \\
\hline $\mathbf{S}$ & 38.7 & .3 & 38.3 & .2 & 38.2 & .2 & 38.1 & .3 & 38.1 & .2 & 37.8 & .2 \\
\hline \multicolumn{13}{|c|}{ Week 2} \\
\hline $\mathbf{P}$ & 38.4 & .2 & 38.2 & .2 & 38.2 & .1 & 37.9 & .2 & 38.1 & .2 & 37.8 & .2 \\
\hline $\mathbf{S}$ & 38.6 & .2 & 38.1 & .3 & 38.0 & .3 & 37.9 & .3 & 37.8 & .3 & 37.7 & .3 \\
\hline \multicolumn{13}{|c|}{ Week 3} \\
\hline $\mathbf{P}$ & 38.0 & .2 & 37.9 & .2 & 37.6 & .2 & 37.8 & .2 & 37.9 & .1 & 37.6 & .2 \\
\hline$S$ & 38.5 & .2 & 37.8 & .2 & 37.9 & .2 & 37.6 & .2 & 37.7 & .3 & 37.6 & .3 \\
\hline
\end{tabular}

evidence for tolerance to the pyrexic effects of poly I:C. It should be noted that there were a number of other lowerlevel interactions significant in the analysis; however, these interactions provided no theoretically important information beyond that contained in the highest order interaction.

\section{Analysis of Raw data}

The raw temperature data were also analyzed with essentially the same interpretation as the transformed delta scores. Group means of raw data for Phases 1 and 2 can be seen in Tables 1 and 2, respectively.

Phase 1. In Phase 1, the analysis of baseline temperature indicated that temperatures decreased as a function of weekly injection $[F(2,42)=4.14, p<.05]$. Temper- ature for the six hourly measurements indicated that temperature decreased both over weeks and over hours following injection. As would be expected, the latter decline was more precipitous for the mice that received the placebo than for those that received the drug, as revealed by a group $\times$ blocks $\times$ hours interaction $[F(2,43)=8.57$, $p<.01]$. The raw data in Phase 1 , then, reveal modest but significant changes in temperature as a function of the drug, and these differences were most visible 2-4 h postinjection. Furthermore, overall decreases in temperature as a function of repeated injection and temperature assessment were observed.

Phase 2. The analysis of the Phase-2 raw temperature data was also highly consistent with the pattern of the transformed data. The major result was a significant in-

Table 2

Group Mean Temperature \pm Standard Error of the Mean for Experiment 2, Phase 2

\begin{tabular}{|c|c|c|c|c|c|c|c|c|c|c|c|c|}
\hline \multirow[b]{3}{*}{ Group } & \multicolumn{12}{|c|}{ Hour } \\
\hline & \multicolumn{2}{|c|}{1} & \multicolumn{2}{|c|}{2} & \multicolumn{2}{|c|}{3} & \multicolumn{2}{|c|}{4} & \multicolumn{2}{|c|}{5} & \multicolumn{2}{|c|}{6} \\
\hline & $M$ & $S E M$ & $M$ & $S E M$ & $M$ & $S E M$ & $M$ & $S E M$ & $M$ & SEM & $M$ & $S E M$ \\
\hline \multicolumn{13}{|c|}{ Week 1} \\
\hline P-S & 38.6 & .2 & 38.1 & .2 & 37.8 & .4 & 38.0 & .3 & 37.8 & .3 & 37.9 & .3 \\
\hline S-S & 38.8 & 3 & 38.5 & .3 & 38.3 & .3 & 38.2 & .4 & 38.5 & .3 & 38.3 & .4 \\
\hline \multicolumn{13}{|c|}{ Week 2} \\
\hline P-S & 38.6 & .2 & 37.9 & .3 & 38.0 & .2 & 37.5 & .3 & 37.7 & .3 & 37.9 & .3 \\
\hline S-S & 38.8 & .2 & 38.5 & .2 & 38.5 & .4 & 38.1 & .4 & 37.8 & .5 & 38.1 & .4 \\
\hline \multicolumn{13}{|c|}{ Week 3} \\
\hline P-S & 38.8 & .2 & 38.1 & .3 & 37.8 & .2 & 38.0 & .3 & 38.0 & .3 & 37.8 & .3 \\
\hline S-S & 38.7 & .2 & 38.5 & .3 & 38.6 & .3 & 38.5 & .3 & 38.2 & .4 & 38.3 & .4 \\
\hline \multicolumn{13}{|c|}{ Week 4} \\
\hline P-S & 38.6 & .1 & 38.0 & .2 & 37.9 & .2 & 37.7 & .3 & 37.2 & .3 & 37.6 & .3 \\
\hline S-S & 38.8 & .2 & 38.4 & .3 & 38.5 & .3 & 38.2 & .2 & 38.1 & .3 & 38.2 & .3 \\
\hline
\end{tabular}

Note-P-S $=0.2 \mathrm{ml}$ i.p. injection of polyinosinic polycytidylic acid (poly I:C) in Phase 1 and physiological saline in Phase 2; S-S $=0.2 \mathrm{ml}$ i.p. injection of physiological saline in Phases 1 and 2. 
teraction involving groups with hours and blocks $[F(2,38)$ $=6.96, p<.01]$. Once again, the mice in Group P-S, with the exception of the first hour, had significantly lower rectal temperatures relative to the remaining groups. The only difference in the analysis of the raw data was that an interaction of weeks was not observed. On the whole, however, the analysis of the Phase 2 raw data supported the presence of a robust hypothermic response in Group P-S.

\section{DISCUSSION}

As noted in the introductory portion of this report, a Pavlovian conditioning analysis of drug tolerance (Siegel, 1978) was indirectly supported by the modulation of tolerance to poly I:C-induced NK cell activation by nonpharmacological conditioning manipulations (Dyck et al., 1987; Dyck et al., 1986). However, previous inferences about conditioning were based on an altered UR to poly I:C. In the present study, the effects of the identical conditioning protocol led to drug-compensatory alterations in body temperature (i.e., hypothermia). These results are generally consistent with a compensatory conditioning analysis. According to such an analysis, an anticipatory response (CR), which opposes the unconditional effects of the drug (UR), develops to cues paired with the drug. In the present study, the UR to the drug was a modest elevation in rectal temperature (hyperthermia) approximately 3-5 h following injection, whereas the CR was a robust compensatory decrease in temperature to cues previously paired with the drug. Just as the unconditional hyperthermic effect was maximal 3-5 $\mathrm{h}$ postinjection, so too was the hypothermic effect observed in the second phase. Although the observation of a hypothermic response to cues previously paired with poly I:C favors a compensatory conditioning interpretation such as that emphasized by Siegel (1978), a recent variation of this hypothesis, which emphasizes the relationship between the form of the CR and the underlying UR (Paletta \& Wagner, 1986), is not supported by the present data. According to Paletta and Wagner's "dual process" model of drug tolerance, a necessary condition for the development of drug-compensatory CRs is the observation of a biphasic UR. That is, associative tolerance should only reflect a drug-compensatory $C R$ when the drug-elicited UR is characterized by a compensatory secondary phase. In this analysis, in the absence of such a biphasic UR, any associative tolerance would be only a consequence of the tendency for the conditioned diminution of the UR (see Wagner, 1981). In the present experiments, the UR was a monophasic hyperthermic response, but this did not preclude the observation of a compensatory hypothermic response to cues previously paired with the drug. It should be acknowledged, however, that with a higher drug dose and a greater number of subjects it might have been possible to demonstrate a compensatory secondary component to the poly I:C-induced pyrexic response.
Although the present results are consistent with a conditioning analysis, the evidence is not conclusive. For one thing, the design did not include a cue manipulation in Phase 2. It is, therefore, not known whether the hypothermic response evidenced by Group P-S was specific to the cues previously paired with the drug. A second problem concerns the continuing robust nature of the hypothermic effect following several extinction trials. It is conceivable that the CR (hypothermia) did not receive a sufficient number of extinction trials to attenuate, and the durability of the response may indicate a potent conditioning procedure, not unlike the taste-aversion paradigm (Ader, 1976; Ader \& Cohen, 1975; Ader, Cohen, \& Bovbjerg, 1982; Ader, Cohen, \& Grota, 1979; Bovbjerg, Ader, \& Cohen, 1984; Kloserhalfen \& Klosterhalfen, 1983). However, without evidence of extinction of the response, it is also possible that the hypothermia may not be a conditioning phenomenon. Given these caveats, in the future it will be necessary to evaluate systematically the context specificity of the hypothermic effect and its sensitivity to extinction manipulations.

A brief comment on the possible importance of the present results for identifying a neuroimmunoregulatory pathway seems in order. It was previously observed that associative factors contribute to poly $\mathrm{I}: \mathrm{C}$-induced tolerance of NK cell activation. Utilizing a similar conditioning protocol, the present results suggest that the effects of poly I:C stimulation on the central nervous system, as measured by thermoregulatory changes, are also conditionable. Although the source of the pyrexic effect was not identified, we would hypothesize that this effect occurs via the release of IL-1 from macrophages stimulated by poly I:C. Such a hypothesis would be consistent with other recently reported central effects of IL-1 on neuropeptide release and corticosterone production (Besedovsky et al., 1986), which, through their immunosuppressive properties, quite possibly contribute to the NK cell tolerance phenomenon.

\section{REFERENCES}

ADER, R. (1976). Conditioned adrenocortical steroid elevations in the rat. Journal of Comparative \& Physiological Psychology, 90, 1156-1163.

ADER, R., CoHEN, N. (1975). Behaviorally conditioned immunosuppression. Psychosomatic Medicine, 37, 333-340.

Ader, R., Cohen, N., Bovbjerg, D. (1982). Conditioned suppression of humoral immunity in the rat. Joumal of Comparative \& Physiological Psychology, 96, 517-521.

Ader, R., Cohen, N., \& Gota, L. J. (1979). Adrenal involvement in conditioned immunosuppression. International Journal of Immunopharmacology, 1, 141-145.

Baker, T. B., \& TIFfany, S. T. (1985). Morphine tolerance as habituation. Psychological Review, 92, 78-108.

Besedovsky, H., del Rey, A., Sorkin, E., Dinarello, C. A. (1986). Immunoregulatory feedback between interleukin-1 and glucocorticoid hormones. Science, 233, 652-654.

Bovbjerg, D., Ader, R., \& Cohen, N. (1984). Acquisition and extinction of conditioned suppression of a graft-vs-host response in the rat. Journal of Immunology, 132, 111-113. 
Dinarello, C. A. (1984). Interleukin-1. Review of Infectious Diseases, 6, 51-72.

Dinarello, C. A., Cannon, J. G., Walff, S. M., Bernheim, H., Beutler, G., Cerami, A., Figari, I., Palladino, M., \& O'ConNER, J. (1986). Tumor necrosis factor (cachectin) is an endogenous pyrogen and induces the production of interleukin-1. Journal of Experimental Medicine, 163, 1433-1450.

Dinarello, C. A., Conti, P., \& Weir, J. (1986). Effects of human interleukin-1 on natural killer cell activity: Is fever a host defense mechanism for tumor killing? Yale Journal of Biological Medicine, $59,97-106$.

Dyck, D. G., Driedger, S. M., Nemeth, R., Osachuk, T. A. G., \& GREENBERG, A. H. (1987). Conditioned tolerance to drug-induced (poly I:C) natural killer cell activation: Effects of drug-dosage and context-specificity parameters. Brain, Behavior \& Immunity, 1, 251-266.

Dyck, D. G., Greenberg, A. H., \& Osachuk, T. A. G. (1986). Tolerance to drug-induced (poly I:C) natural killer cell activation: Congruence with a Pavlovian conditioning paradigm. Journal of Experimental Psychology: Animal Behavior Processes, 12, 25-31.

KIRK, R. E. (1968). Experimental design: Procedures for the behavioral sciences. Belmont, CA: Wadsworth.

Klosterhalfen, W., \& Klosterhalfen, S. (1983). Pavlovian conditioning of immunosuppression modifies adjuvant arthritis in rats. Behavioral Neuroscience, 97, 663-666.

PalettA, M. S., \& WAgner, A. R. (1986). Development of context specific tolerance to morphine: Support for a dual process interpretation. Behavioral Neuroscience, 100, 611-623.

SIEGEL, S. (1978). Tolerance to the hypothermic effect of morphine in the rat is a learned response. Journal of Comparative \& Physiological Psychology, 92, 1137-1149.

WAGNER, A. R. (1981). SOP: A model of automatic memory processing in animal behavior. In N. E. Spear \& R. R. Miller (Eds.), Information processing in animals: Memory mechanisms (pp. 547). Hillsdale, NJ: Erlbaum.

\section{NOTE}

1. Although we realize that presenting the data in its raw form may be more appropriate, using delta scores reduces the complexity of interpretation both visually and statistically. Furthermore, we have explored the data in both its raw and its transformed state and found essentially no differences in interpretation. For these reasons, we prefer to focus on the delta scores to facilitate presentation and interpretation of the data to the reader. However, where appropriate (e.g., Experiment 2), both raw and transformed data will be presented to show the minimal differences in interpretation by the two methods of data presentation.

(Manuscript received December 30, 1987; revision accepted for publication January $3,1989$. ) 